\title{
Location planning of electric taxi charging station based on driving trajectory
}

\author{
Hehuan $^{1, a^{*}}, \mathrm{GeQin}^{2, \mathrm{~b}}$ \\ ${ }^{1}$ Department of Economic and Management, Jiangsu University of Science and Technology, Zhenjiang, Jiangsu, 212000, China \\ ${ }^{2}$ Department of Economic and Management, Jiangsu University of Science and Technology, Zhenjiang, Jiangsu, 212000, China
}

\begin{abstract}
With the vigorous development of energy technology, the promotion and use of electric vehicles has brought new vitality to the development of ecological environment. According to the big data of taxi travel trajectory, we can get the idle rule of taxi operation time. Taking the idle time and location of taxi as the demand hot spot, taking the location points along the road network of the research area as the candidate points, this paper uses ArcGIS to do the location planning of charging station, so that the charging station can reduce the charging anxiety of taxi drivers, meet the charging demand to the maximum extent, and get the the minimum number of stations, and the quantity and distribution are taken as the site selection scheme..
\end{abstract}

\section{Introduction}

With the rapid development of society, the consumption of oil resources is intensified, and the problem of environmental pollution is becoming more and more serious. Electric vehicles with its zero exhaust emissions, low noise pollution and other good environmental benefits and social benefits have been widely concerned, which promotes the development of new energy vehicles. The development of electric vehicle industry not only injects strong new energy into the economic growth of various countries, but also makes great contribution to reducing greenhouse gas emissions, coping with the challenge of climate change and improving the global ecological environment.Although the public acceptance of EVs is gradually increasing, the situation of EVcharging has not fundamentally changed, the dilemmas of charging difficulty and low utilization rate of charging facilities are still prominent. On the one hand, the existed public charging facilities can' $t$ meet the charging demand of electric vehicles, the charging infrastructures need vigorous construction. On the other hand, there are a large number of idle charging piles, which can' $t$ be conveniently used by EVs drivers. All of which hinder the further promotion and popularization of EVs to a certain extent.

By September 2020, China has built 42000 charging stations, 525 switching stations, 1.42 million various charging piles, and the vehicle to pile ratio is about 3.1:1. Data source: issued by the Ministry of industry and information technology at the policy meeting of the State Council in November 2020. There is still a long way to go from the 1:1 construction goal. It can be seen that at present, the number of public charging facilities can not meet the charging demand of electric vehicles. The phenomenon of "easy to buy a car, difficult to charge" is widespread in the actual needs of the public, which hinders the promotion and popularization of electric vehicles to a certain extent.

With the vigorous development of energy technology, a new round of scientific and technological revolution and industrial change is gradually rising. The new energy vehicle industry has entered a new stage of accelerated development. Vigorously developing new energy vehicles has become a breakthrough to solve many problems, and also an inevitable choice for the transformation and upgrading of China's automobile industry structure. The development of new energy vehicles has been upgraded to a national strategy. In order to guide consumers to choose new energy vehicles, the state has also promulgated a series of incentive policies.

\section{Literature review}

At present, the main research focuses on the number, address and capacity of charging stations.

In terms of location objectives, $\operatorname{Sun}^{[1]}$ et al. Established the maximum coverage model and multiobjective programming. $\mathrm{Ye}^{[2]}$ et al. established a model to minimize the system travel time and greenhouse gas emissions, considering that the road impedance in the traffic problem will affect the route selection of new energy vehicles, which makes the research on the charging demand of new energy vehicles more consistent with the actual situation.Zeng ${ }^{[3]}$ et al. used Markov chain to forecast the demand, established a mathematical model to maximize the revenue of charging station, and analyzed the optimal solution with immune optimization algorithm. $\mathrm{Li}^{[4]}$ control the cost from two aspects of user use cost and construction cost, and propose bacterial colony optimization algorithm to

a Corresponding author: hehuan_7@163.com 
find the optimal solution. Zeinab ${ }^{[5]}$ introduces a network of charging stations with various charging options. The goal is to determine a charging station that ensures the minimum charging time, driving time and charging cost.

The research focus of charging station planning is to minimize costs and maximize coverage. But because of the lack of a large number of track and time information, most of the demand forecasting can not get the exact time of a driver to arrive at a certain location and stay, so it is impossible to judge the charging intention and charging possibility of electric vehicle drivers. In order to solve this problem, this paper analyzes the real taxi GPS data in Chengdu, uses massive mobile location data to extract the potential traffic demand location, extracts the points on the road network as the candidate location, and selects the location scheme with the least number of charging stations to cover all the demand as the candidate location of electric vehicle charging station.

\section{Model construction and solution based on ArcGIS}

Collection coverage problem is to find the least number of subsets from facility point set $\mathrm{N}$ to cover all demand points. The model can be solved by ArcGIS software,It needs to be given enough candidate location, demand point and specified service distance.

When ArcGIS is running, the restricted direction of road should be considered. as shown in Figure 1.As shown in the figure 1, Because there are candidate points and demand points that are not on the road network, ArcGIS moves them to the nearest road network when running the calculation, and the distance involved in the calculation is the length of the road network.

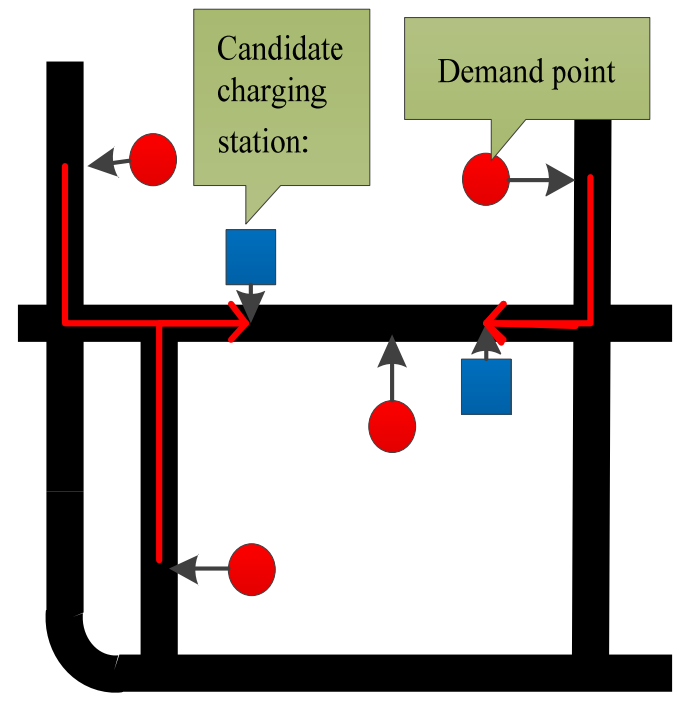

Figure 1. Legend of demand point preferred facility point

The model can be solved by ArcGIS software and location allocation tool of GIS software:

The first step is to generate the start destination matrix of the shortest path cost between all candidate points and demand points, and construct the edited version of the cost matrix through the hillsman process;
In the second step, the location assignment solver generates a set of semi random solutions, and then optimizes these solutions by using the break point replacement heuristic (Teitz Bart).

In this way, a set of effective solutions can be obtained, and on this basis, the meta heuristic algorithm will merge the effective solutions to create better solutions.

If a better solution is not found, it returns to finding the best solution among the effective solutions. The combination of edited matrix, semi random initial solution, break point replacement heuristic algorithm and optimized meta heuristic algorithm are built-in tools for ArcGIS to solve the location allocation problem.

\section{Empirical analysis}

\subsection{Analysis of research area}

Taking Chengdu as an example, ArcGIS is used to visualize the road network map of the study area in Chengdu, as shown in Figure 2.

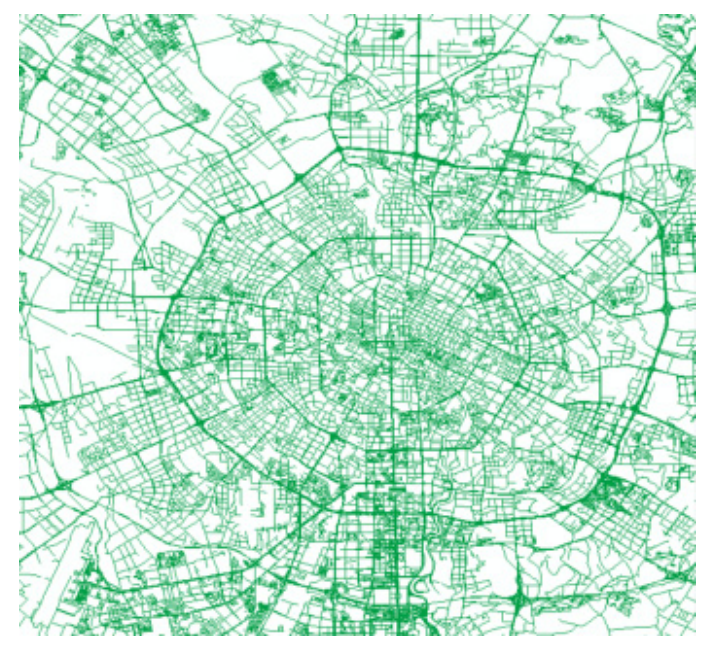

Figure 2. Roadmap network of Chengdu city

The longitude and latitude regions of the road network map in this research are longitude 103.933 104.171, latitude $30.557 \sim 30.776$, which covers most of the central area of Chengdu City, about 520 square kilometers. The road network in the figure contains all the roads and their detailed information within the latitude and longitude range, including highway name, highway number, restricted direction and other attributes. It can be seen from the road network map that the roads in Chengdu are relatively dense in the city center, and the road network distribution is gradually sparse when it spreads around the center.In this paper, from the driver's point of view, according to the low probability period of carrying passengers, that is, the period with more frequent occurrence of idle time, as the best charging period, we find the location of the empty car as the charging demand point in the best charging period. In order to find out the best charging time with high empty rate, this paper uses the number of empty cars with 
continuous empty time more than 60 minutes as the criterion of empty rate.

\subsection{Cleaning and analysis of trajectory big data}

\subsubsection{Formatting Cleaning of trajectory big data}

The taxi track data is from the GPS records from August 3 to August 23, 2014 provided by DC big data competition. The records include the driving tracks of more than 13000 taxis in Chengdu. The driving data are distributed between 6:00 a.m. and 12:00 p.m., including vehicle serial number, passenger carrying status, latitude and time elements. Because the amount of data is too cumbersome, after eliminating the abnormal data and duplicate data, the data is diluted to one data per minute. Due to the discontinuity in the original data, this research selected 5-day complete and continuous data from the original data set, that is, August 18 (Monday) to August 22 (Friday).

In the original data, because of the technical problems when GPS collects information, there are outliers or null values. Firstly, the original data is processed by removing outliers and null values. The key of data arrangement is to remove invalid data. The steps are as follows:

Delete the null value record, that is, remove the record or coordinate that lacks time.

Merge duplicate records into a new record.

Remove invalid data generated by the influence of communication technology.

Due to the large amount and miscellaneous of original data, the interval between longitude and latitude data returned by GPS varies from 2 seconds to 30 seconds. In this research, the GPS return interval data is cleaned to collect position data every minute.

\subsubsection{Analysis of trajectory big data}

As the service group of the charging station is taxi drivers, the business pressure of taxi drivers should be considered when selecting the charging demand point, and the starting point of no-load in the period of high idle probability should be selected as the location where taxi drivers need to charge. Through the extraction of data by SPSS, the most idle time period from 11:00 to 13:00 in five consecutive days is obtained. The starting position of all idle time periods is shown in Figure 3.

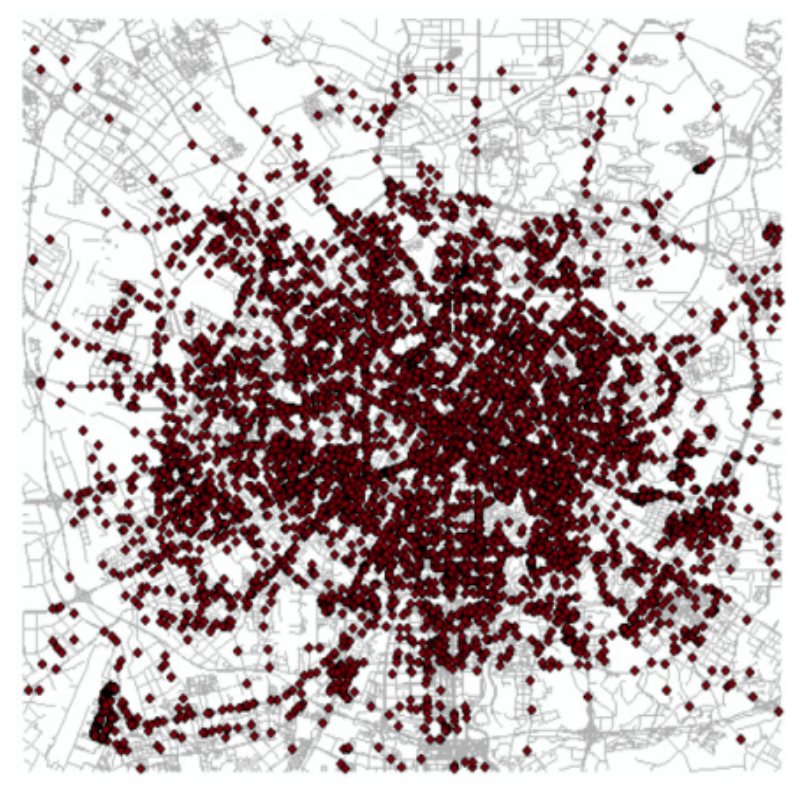

Figure 3. Starting point of idle time for 5 consecutive days

\subsection{Charging station location based on data analysis}

The candidate location of charging station and the location of road network are extracted by ArcGIS. In this study, the location points at the intersection of road sections and every 100 meters from the beginning of each road section are used as candidate locations, so that the location and number of candidate points can be close to the continuous location, and 89858 candidate locations are obtained.As shown in Figure 4.

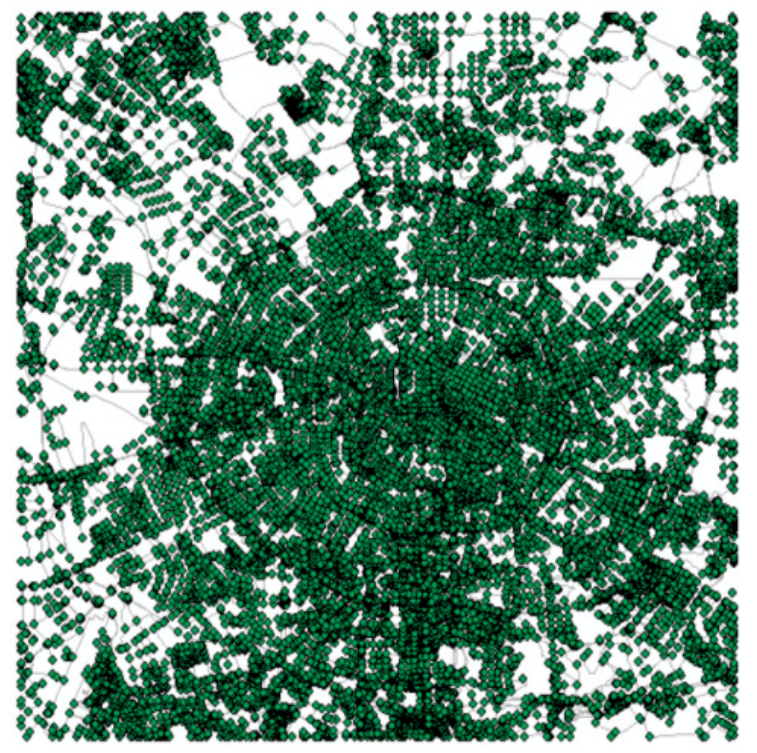

Figure 4. Candidate location in road network

State Grid of China has set the target that the service radius of suburban charging stations should not exceed $5 \mathrm{~km}$, that of peripheral charging stations should not exceed $3 \mathrm{~km}$, and that of urban charging stations should not exceed $1 \mathrm{~km}$. Chengdu is a demonstration and promotion area for electric vehicles. According to the target planning of State Grid, the radius of public 
charging service in Chengdu urban core area should be less than $1 \mathrm{~km}$. Taking $1 \mathrm{~km}$ as the service radius, the demand points are divided in strict accordance with the driving direction from the demand point to the candidate point and the route. Here, according to the minimum number of facilities, that is, the set coverage model, the minimum number of facilities can cover the most demand points. After ArcGIS calculation, the planning results are shown in Figure 5. The green dots in the figure represent the location of the site, and the number is 409 .

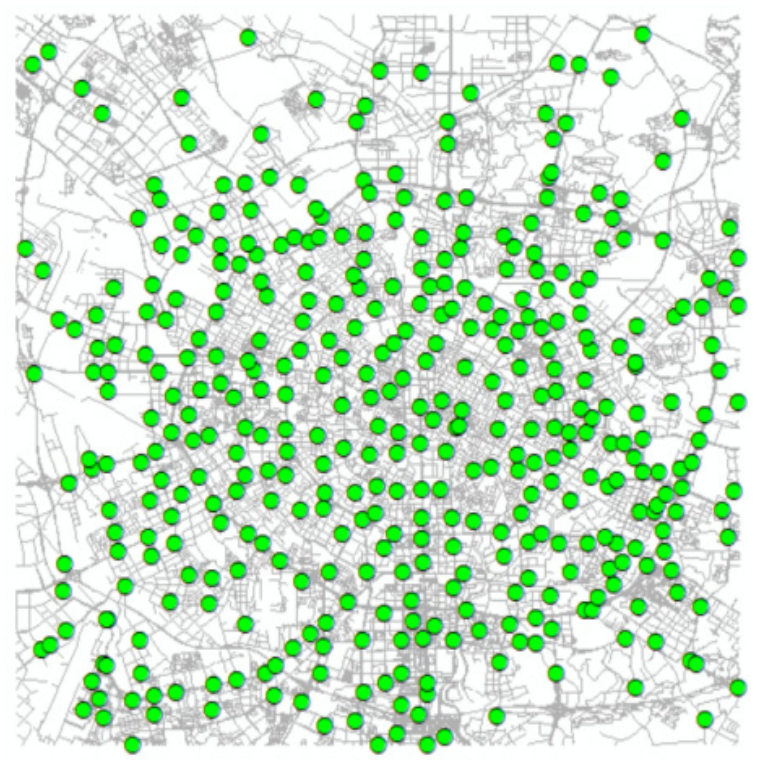

Figure 5. Selected charging station location

\section{Conclusions}

In this paper, The full use of big data makes the planning of electric taxi charging station more reasonable. Using ArcGIS to analyze the road network,more than 80000 candidate locations are obtained, which makes the charging station location problem closer to the continuous location problem, and improves the accuracy of charging station location. ArcGIS software is used to select the location of charging stations through spatial analysis of driving track points. Based on the social benefits of charging stations, the number and location of charging stations are reasonably planned.

\section{References}

1. Zhuo Sun; Wei Gao; Bin Li; Longlong Wang.Locating charging stations for electric vehicles.J. Transport Policy, 0967-070X.(2018)

2. Ye Lu, Guo Qianyun, Ni Shuchen, Feng Chun.Location model of charging station in hybrid transportation network.J.17(04):97-104.(2019)

3. Zeng Chengbi, Liu Guang, Miao Hong, Wang Ya, Han Feng, Cheng Fanfan.Electric vehicle charging station planning considering urban traffic flow.J.Journal of Electric Power Science and Technology, 34 (04): 101-107. (2019)
4. Li Yuanbo, Jiang Tiezheng, Chen Jiajun.Multi objective programming of electric vehicle charging station based on super efficiency DEA.J.Power technology,40(04): 849-860.(2016)

5. Zeinab Moghaddam; Iftekhar Ahmad; Daryoush Habibi; Quoc Viet Phung.Smart Charging Strategy for Electric Vehicle Charging Stations.J. IEEE Transactionson Transportation Electrification, 4(1): 76-88.(2018) 